\title{
Expanding Dietary Therapy Beyond the Classic Ketogenic Diet: On the Use of the Modified Atkins Diet and Low Glycemic Index Treatment in Pediatric Epilepsy
}

\author{
Robyn Whitney, RaJesh RaMachandRan NAIR \\ Comprehensive Epilepsy Program, Division of Neurology, Department of Paediatrics, McMaster University, \\ Hamilton, ON, Canada. \\ Correspondence to: Rajesh Ramachandran Nair, Comprehensive Epilepsy Program, McMaster Children's \\ Hospital McMaster University, Hamilton, Ontario, Canada.rnair@mcmaster.ca
}

1 pproximately one third of children with epilepsy are deemed medically refractory. Non-pharmacological interventions, such as dietary therapies, provide an opportunity to achieve seizure control, in those whose seizures cannot be controlled by anti-seizure medications (ASMs) and are not candidates for epilepsy surgery. A variety of forms of dietary therapy are available, including the classic ketogenic diet (KD), medium-chain triglyceride $\operatorname{diet}(\mathrm{MCT})$, the modified Atkins diet (MAD) and the low glycemic index treatment (LGIT) [1]. The KD may be difficult to adhere to for some patients, due to a variety of reasons such as cost, ease of administration, palatability, and side effect profile. Recently, there has been increasing interest in less restrictive forms of dietary therapy, such as the MAD and LGIT with the goal of improving compliance and with the benefit of maintaining seizure control [1-10].

Although the benefits of individual dietary therapies have been documented, studies comparing the effectiveness of more liberal forms of diet therapy (i.e. MAD, LGIT) are lacking [10]. In addition, there is a paucity of studies comparing the effectiveness of the MAD and LGIT to the classic KD [10]. However, recently, an RCT from India compared the efficacy of the MAD, KD and LGIT and demonstrated a similar median reduction in seizure burden between all three diets. Further, neither the MAD or LGIT met noninferiority criteria when compared to the KD [10]. In this issue of Indian Pediatrics, Gupta, et al. [11] compare the effectiveness of the MAD and LGIT diet among children with medically refractory epilepsy. The authors assert that although the MAD is more liberal than the classic KD, it may have drawbacks with compliance and that LGIT may be viewed as more palatable with a milder side effect profile, and also provide the benefit of seizure control. The authors compared the effectiveness of the MAD and LGIT with the hypothesis that there would be no difference in seizure control between the two therapies [11].

In this open label RCT, children aged 6 months to 14 years with medically refractory epilepsy were randomized to either the MAD or LGIT as add on therapy and were followed for a total of 12 weeks [11]. The primary outcome was the proportion of children achieving seizure freedom at 12 weeks [11]. Secondary outcomes included the proportion of children who achieved $>50 \%$ and $>90 \%$ seizure reduction at 12 weeks [11]. Gupta, et al. [11] demonstrate that in the short-term, seizure freedom and $90 \%$ seizure reduction rates are similar between the MAD and LGIT. Albeit, few children on either diet obtained seizure freedom, which is not uncommon with dietary therapy [4-8]. Despite this, both therapies provided benefit with a substantial portion of patients achieving $50-90 \%$ seizure reduction [11]. At 12 weeks, there was some benefit in achieving 50-90\% seizure reduction with LGIT, although this finding should be interpreted with caution given the small case counts and effect size [11]. Larger studies are therefore needed to replicate these findings. When compared to previous studies, the proportion of patients achieving at least a $50 \%$ seizure reduction with LGIT was higher at 3 months, while the effectiveness of the MAD was lower [4,6,7-9]. Moreover, the number of patients achieving at least $50 \%$ seizure reduction at 1 month with LGIT was lower than previous reports [8]. A drop in efficacy of the MAD between 1 month and 3 months was also reported. The reason for the drop in efficacy of the MAD between 1 and 3 months, is unknown. Compliance was reported to be sustained throughout the study, although could conceivably result in this decrease. The authors claim that the higher response with LGIT at 3 months may be due to the fact that previous studies were conducted in adults and patients with tuberous sclerosis complex. Although, other pediatric cohorts of LGIT have documented lower 
rates of patients achieving $>50 \%$ seizure reduction at 3 months (i.e. 30-50\%) [8,9].

The etiologies of epilepsy were not clear in the present study, nor were the spectrum of epilepsy syndromes, which was a drawback of the study (some had Lennox Gastaut syndrome and West syndrome). Neonatal problems were documented, although it is unclear, whether these conditions were responsible for the epilepsy. This information would have been helpful to determine if certain epilepsy syndromes/etiologies respond better to either diet. A shortcoming of the study was the lack of long-term follow up. Longer term studies are needed to determine the comparable efficacy of the diet, and the authors do acknowledge this. Further, the median age of patients in each group was young (i.e. 24-30 months) and the generalizability of the results to older children is unclear. Only five patients across the study were lost to follow-up and no children appeared to withdraw from the study secondary to adverse events, which was a strength of the study. Although longer term studies are needed to determine whether compliance as well as tolerability are sustained, given that these are reported benefits of the diets. The inclusion of children with normal development in future studies would be important. Further, future studies should also address the effects of both diets on cognition and quality of life.

Overall, the authors should be commended for their study. Their work further emphasizes the need to consider the spectrum of dietary therapies when encountering patients with refractory epilepsy. An individualized approach, which considers a myriad of factors when prescribing dietary therapy, is important [10].

Contributors: Both authors have made substantial contributions to all aspects of the project.

Funding: None. Competing interests: None stated.

\section{REFERENCES}

1. Kossoff EH, Hartman A. Ketogenic diet: New advances for metabolism based therapies. Curr Opin Neurol. 2012;25:173-78

2. Gauthier A, Simic N, Jones KC, Ramachandran Nair R. Modified Atkins diet with slow reduction of carbohydrate. Epilepsy Behav Rep. 2019: 13:100353.

3. Kossoff EH, Dorward JL. The modified Atkins diet. Epilepsia. 2008; 49(suppl 8):37-41.

4. Miranda MJ, Mortensen M, Povlsen JH, Nielsen $\mathrm{H}$, Beniczky S. Danish study of a modified atkins diet for medically intractable epilepsy in children: Can we achieve the same results as with the classic ketogenic diet? Seizure. 2011;20:151-55.

5. Chen W, Kossoff EH. Long-term follow-up of children treated with the modified Atkins diet. J Child Neurol. 2012; 27:754-58.

6. Kossoff E, McGrogan JR, Bluml RM, Pillas DJ, Rubenstein JE, Vining EP. A modified Atkins diet is effective for the treatment of intractable pediatric epilepsy. Epilepsia. 2006;47:421-24.

7. Kang HC, Lee HS, You SJ, Kang DC, Ko TS, Kim HD. Use of a modified Atkins diet in intractable childhood epilepsy. Epilepsia. 2007;48:182-86.

8. Muzykewicz DA, Lyczkowski DA, Memon N, Conant KD, Pfeifer HH, Thiele EA. Efficacy, safety, and tolerability of the low glycemic index treatment in pediatric epilepsy. Epilepsia. 2009;50:118-26.

9. Lakshminarayanan K, Agarawal A, Panda PK, et al. Efficacy of low glycemic index diet therapy (LGIT) in children aged 2-8 years with drug resistant epilepsy: A randomized control trial. Epilepsy Res. 2021;171:106574.

10. Sondhl V, Agarwala A, Pandley R, et al. Efficacy of ketogenic diet, modified atkins diet and low glycemic index therapy diet among children with drug-resistant epilepsy: A randomized control trial. JAMA Pediatr. 2020;174:944-51.

11. Gupta S, Dabla S, Kaushik JS. Modified Atkins diet versus low glycemic index treatment for drug-resistant epilepsy in children: An open label, randomized control trial. Indian Pediatr. 2021 Feb 25; S097475591600297. 